\title{
Ionospheric anomaly due to seismic activities - Part 2: Evidence from D-layer preparation and disappearance times
}

\author{
S. K. Chakrabarti ${ }^{1,2}$, S. Sasmal ${ }^{2}$, and S. Chakrabarti ${ }^{2,3}$ \\ ${ }^{1}$ S. N. Bose National Centre for Basic Sciences, JD Block, Salt Lake, Kolkata 700098, India \\ ${ }^{2}$ Indian Centre for Space Physics, 43 Chalantika, Garia Station Road, Kolkata 700084, India \\ ${ }^{3}$ Maharaja Manindra Chandra College, 20, Ramkanta Bose Street, Kolkata 700003, India
}

Received: 2 November 2009 - Revised: 11 May 2010 - Accepted: 13 July 2010 - Published: 25 August 2010

\begin{abstract}
We show evidences for anomalous ionospheric behaviour in the signal of Indian navy VLF transmitting station named VTX due to earthquakes in the South Asian region. We concentrate on the variation of the D-layer preparation time (DLPT) and D-layer disappearance time (DLDT) in a period of sixteen months and study their average behaviors. We identify those days in which DLPT and DLDT exhibit significant deviations. Separately, we compute the energy release by earthquakes during this period and show that "anomalous VLF" days are associated with anomalous energy release. We find that the anomaly and the deviation of DLPT and DLDTs from the mean are linearly correlated. We discuss the predictability in this approach and compare with the terminator shift approach using the same set of data.
\end{abstract}

\section{Introduction}

In Sasmal and Chakrabarti (2009, hereafter Paper 1), the behaviour of the signal from the Indian Navy station VTX (transmitting at $18.2 \mathrm{KHz}$ ), especially the behaviour of the terminators, as received at Kolkata, was presented. Data of four solar quiet years from 2005 to 2008 were used to obtain the averaging. From signals received on seismically active days, we specifically studied the behaviours of sunrise and sunset terminators, and found that there is a higher possibility of detecting anomalous terminator shifts typically two days prior to actual earthquakes. That the sunrise and sun-set terminators exhibit shifts towards night before earthquakes were known quite a while (Molchanov et

Correspondence to: S. K. Chakrabarti (chakraba@bose.res.in) al., 1998; Molchanov and Hayakawa, 1998; Hayakawa and Molchanov, 2000; Hayakawa et al., 2003; Chakrabarti et al., 2005; Maekawa et al., 2006; Rozhnoi et al., 2009). Prior to this approach, workers such as Gokhberg et al. (1989) and Gufeld et al. (1992) showed that several days before the earthquake there were anomalies in the nighttime radio signal. However, the relationship between the anomalous terminator times (or any other anomaly for that matter) and seismicity, (which could be due to a combination of lithosphericionospheric coupling, sono-luminescence, breaking of bonds in tectonic plates, etc.) is poorly understood as of now (e.g., Rodger et al., 1999; Hayakawa et al., 2003). Some theoretical models have been advanced and numerical simulations were carried out. It was found using simple models that the lowering of the ionosphere by $1-2 \mathrm{~km}$ could mimic the terminator time shift (Hayakawa et al., 1996; Molchanov et al., 1998) although a more careful and realistic model showed that it would require a lowering of the ionosphere by $4-11 \mathrm{~km}$ which would have other observational effects as happens in the cases of solar flares (Rodger et al., 1999; Soloviev and Hayakawa, 2002; Soloviev et al., 2004). It was concluded that observed shifts in terminators are possible only if the propagation path is short $(<2500-3000 \mathrm{~km})$.

In order to have more input to theoretical studies, we feel that it is essential to look for other types of correlations which may be present. For instance, the time taken to lower the Dlayer boundary in the early morning (we call this as the Dlayer preparation time or DLPT), and the time taken to raise it again in the evening (we call it the D-layer disappearance time) may also be affected by the seismic activities. This is because, in presence of extra ionizing agent, the times taken for such activities may be altered significantly.

Published by Copernicus Publications on behalf of the European Geosciences Union. 


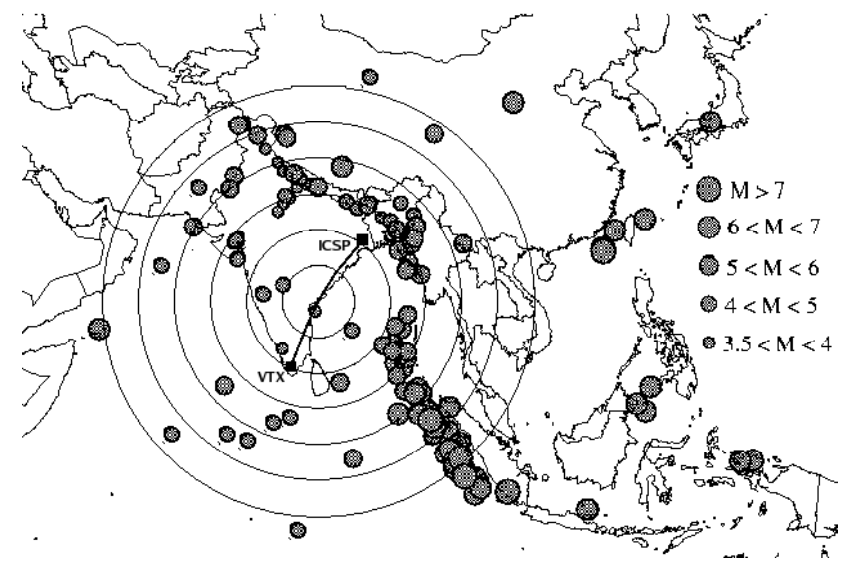

Fig. 1. Location of the earthquakes in relation to seismic circles centered around the first reflection point (FRP) of the VTX signal to ICSP, Kolkata, during the sixteen months under study. The locations of VTX and ICSP are marked with filled boxes and the FRP is marked with a filled circle. Shadowed circles represent the locations of the earthquakes, their sizes being proportional to the individual magnitudes. Concentric circles have radii of multiples of $500 \mathrm{~km}$.

In the present paper, we concentrate on the signal of sixteen months duration (from November 2006 to February 2008) from the Indian Navy operated transmission station VTX (located at Vijayanarayanam, Lat. $08^{\circ} 26^{\prime} \mathrm{E}$, Long. $77^{\circ} 44^{\prime} \mathrm{N}$ ) as received by Standford University made AWESOME receiver with cross-loop antennas and ICSP made Gyrator-II receiver with one loop antenna. The receiving station is at Kolkata (Lat. $22^{\circ} 34^{\prime} \mathrm{E}$, Long. $88^{\circ} 24^{\prime} \mathrm{N}$ ). take a statistics of all the earthquakes in the region during this period and find that a definite correlation exists between the DLDT or DLPT and the effective magnitudes of the earthquakes. We use the Standardized calibration curve for the VTX-ICSP baseline, However, in terms of predictability, the terminator shift method is found to be better, especially that we are discussing short propagation paths.

In Paper 1, details were presented about the receiver and transmitters used in our study and we do not repeat them here. The plan of the present paper is the following: in the next Section, we present the spatial distribution of the earthquakes, and our methodology is to compute the effective magnitude of these quakes at the mid point between the VTX and ICSP. In Sect. 3, we present the DLDT and DLPT values for the period of sixteen months and determine the mean and deviations from the mean of these quantities. We then find the correlation between the effective magnitudes of the earthquakes and the deviations in DLDT and DLPT. We also determine the predictability of the seismic activities using this method, and compare with that obtained from the terminator shift method. Finally, in Sect. 6 we draw our conclusions.

\section{Statistics of seismic activities}

Before we proceed with our results, we wish to present the statistics of the earthquakes in the region for the period of sixteen months (November 2006-February 2008) under consideration. We gather the data, such as the latitude and longitude of the place of the earthquake, magnitude, depth of the epicenter from the web-page of the Indian Meteorological Department (http://www.imd.ernet.in). In Fig. 1, we present a map in which VTX and ICSP are marked with filled squares. We consider the mid-point of the VTX-ICSP great circle path (marked with a filled circle), which is the first reflection point (FRP) at the ionosphere. Since the second and third reflection points are also important for the VTX signal to Kolkata, we expect that earthquakes closer to VTX or ICSP would also affect the possible ionization in the VTX-ICSP path and therefore the VTX signal amplitude. In order to focus our attention on a few earthquakes which might affect the observed signals, we note that the earthquake preparation zone size is about $L \sim 10^{0.43 M} \mathrm{~km}$, where $M$ is the magnitude of the earthquakes. Since the quakes under consideration are of $M<8$, we assume that quakes up to a distance of $3000 \mathrm{~km}$ from the FRP may be influential. Using the FRP point as the center, we draw six concentric seismic circles, having radii in multiples of $500 \mathrm{~km}$ and consider earthquakes which take place in these regions. Shaded circles show the locations in which earthquakes took place and whose magnitudes were used to compute the energy release, their sizes being proportional to the magnitudes of the quakes. In Fig. 2a, we draw histograms of the total number of earthquakes in these seismic circles. We note that the majority of the earthquakes happened between $1000 \mathrm{~km}$ and $1500 \mathrm{~km}$, thus these quakes could influence the ionosphere through the second and third hops. In order to compute the total energy released by the earthquakes which may affect the ionosphere, we use following formulas (Lowrie, 2007): $\log _{10} E=4.4+1.5 M_{\mathrm{S}}$ (for earthquakes with a magnitude $M<5.0$ ), and $\log _{10} E=5.24+1.44 M_{\mathrm{s}}$ (for earthquakes with a magnitude $M>5.0$ ), where, $E-$ energy released in the earthquake in Joule and $<M_{\mathrm{S}}>=$ $-3.2+1.45 M-$ surface wave magnitude. Using these, we will compute the energy released as seen at FRP. We assume that the energy of a quake drops as a cylindrical wave amplitude $\sim 1 / r$. We compute the great circle path from each of these earthquakes to FRP and calculate the effective energy at FRP. We then obtain the effective magnitude $<M_{\mathrm{S}}>$ of the earthquake at FRP by adding contributions from all the earthquakes which take place in a given day and using the above formula. In Fig. 2b, we draw a histogram of the effective magnitudes of the earthquakes as seen from the FRP. The plot peaks at $\langle M>\sim 3-3.5$. The question we wish to answer is: does this effective magnitude correlate with the possible anomalies in the VLF signal and if so, how. Of course, the major contribution to the effective 
(a)
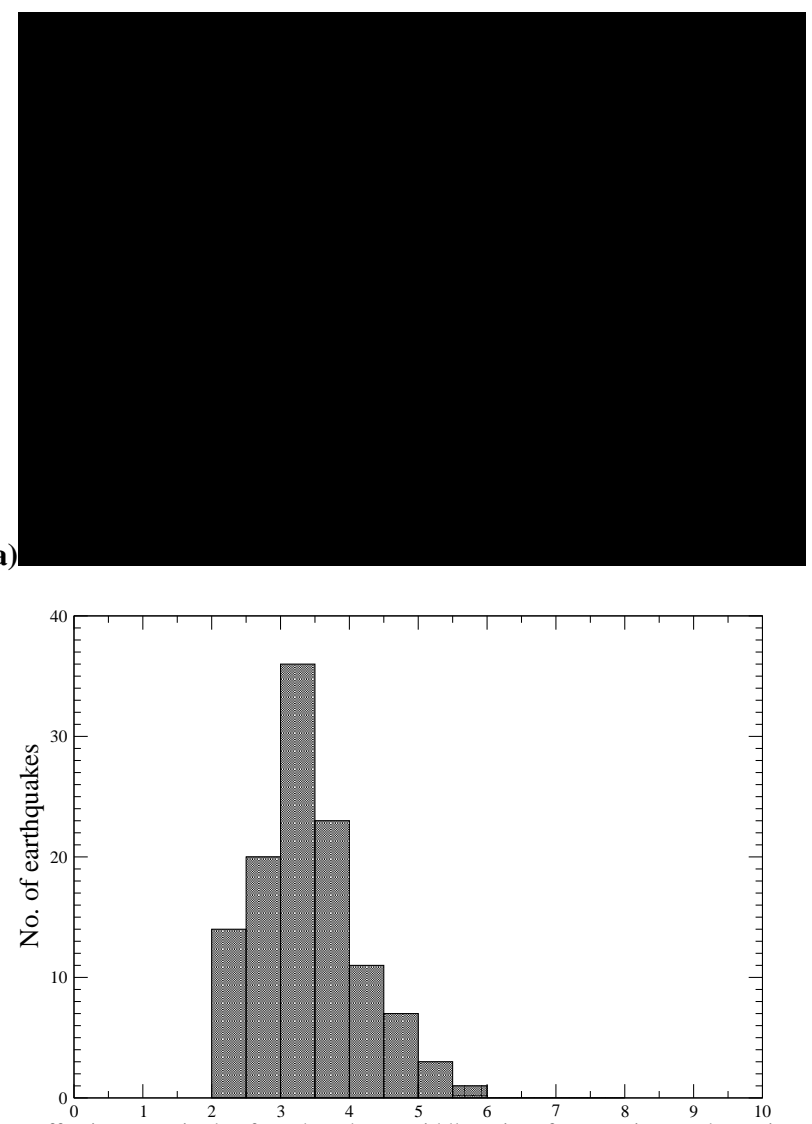

(b) Effective Magnitude of Earthquake at Middle Point of Transmitter and Receiver

Fig. 2. Histograms of (a) total number of earthquakes in the seismic circles showing that the majority of the earthquakes happened between $1000 \mathrm{~km}$ and $1500 \mathrm{~km}$ and (b) the effective magnitudes of earthquakes as seen from the FRP. The plot peaks at $<M_{\mathrm{S}}>\sim 3-3.5$.

energy release is from the strongest quakes. For this reason, we also use the individual magnitudes in a separate analysis and present the results.

\section{The behaviour of DLPT and DLDT}

Following Chakrabarti et al. (2007), we defined two notations in Paper 1, namely, DLPT and DLDT. We define them here again for the sake of completeness. In the dawn, the D-layer boundary takes certain time to go down from its maximum height (Point $T_{\mathrm{A}}$ in Fig. 5 below) to the minimum height (sunrise terminator or SRT, denoted by point $T_{\mathrm{C}}$ in Fig. 5). The difference $T_{\mathrm{C}}-T_{\mathrm{A}}$ is the D-layer preparation time or DLPT. Similarly, at the dusk, the sequence of events is opposite and through points $T_{\mathrm{D}}$, (sunset terminator SST), and points $T_{\mathrm{B}}$, respectively. The difference $T_{\mathrm{B}}-T_{\mathrm{D}}$ is the $\mathrm{D}$ layer disappearing time or DLDT. Out of a total of sixteen months of data that we use, clear data around the sunrise

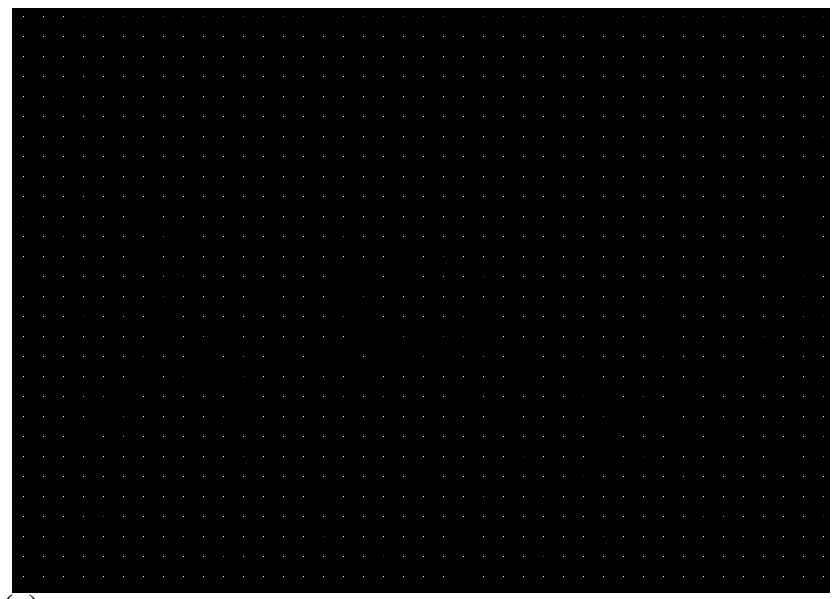

(a)

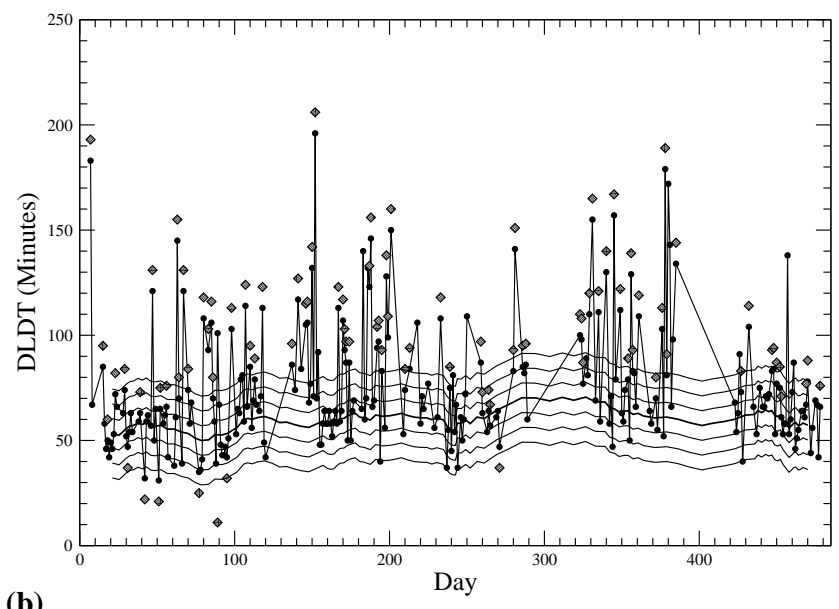

(b)

Fig. 3. Plots of (a) DLPT and (b) DLDT as a function of days. Dark circles are actually observed values of DLPT and DLDT on a given day and the diamonds represent earthquakes which are "associated" with the anomalous data, even when the earthquake may have taken place 2-3 days later. The thick solid curve is the average of the DLPT and DLDT values, and the thin solid curves are drawn at $1 \sigma$, $2 \sigma$ and $3 \sigma$ apart.

were obtained in 300 days, and clear data around the sunset were obtained in 253 days. On the other days, either the transmitter was down or our receiver was down. We compute the DLPT and DLDT and in Fig. 3a-b plotted them as a function of days. Typically, DLPT varies between $30-50 \mathrm{~min}$, the DLDT varies between 50-70 min. However, in a number of days, these values are anomalous. In the figures, dark circles are actually observed values of DLPT and DLDT on a given day, the diamonds represent earthquakes which are "associated" with the anomalous data, even when the earthquake may have taken place 2-3 days later. The thick solid curve is the average of the DLPT and DLDT values, computed by removing days which show anomalies of more than $3 \sigma$. The thin solid curves are drawn at $1 \sigma, 2 \sigma$, and $3 \sigma$ apart. In Fig. $4 a-b$ we show the number of days in which various 
(a)

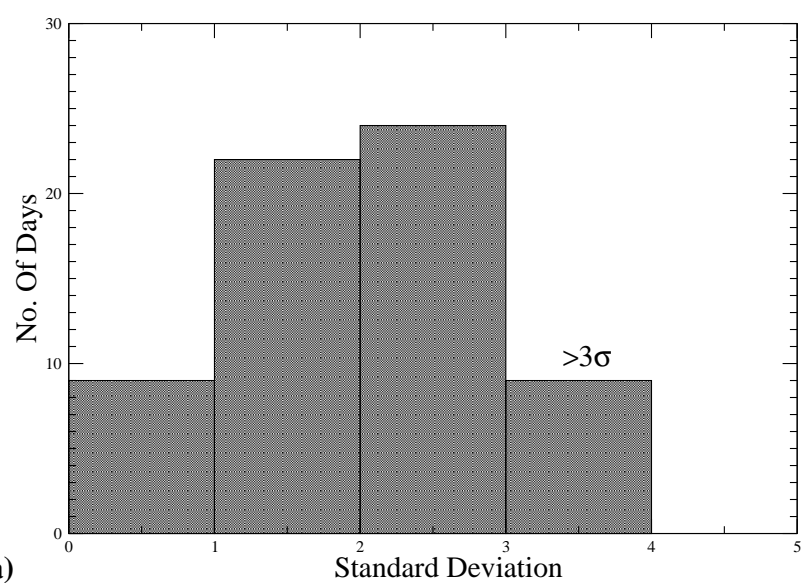

(b)

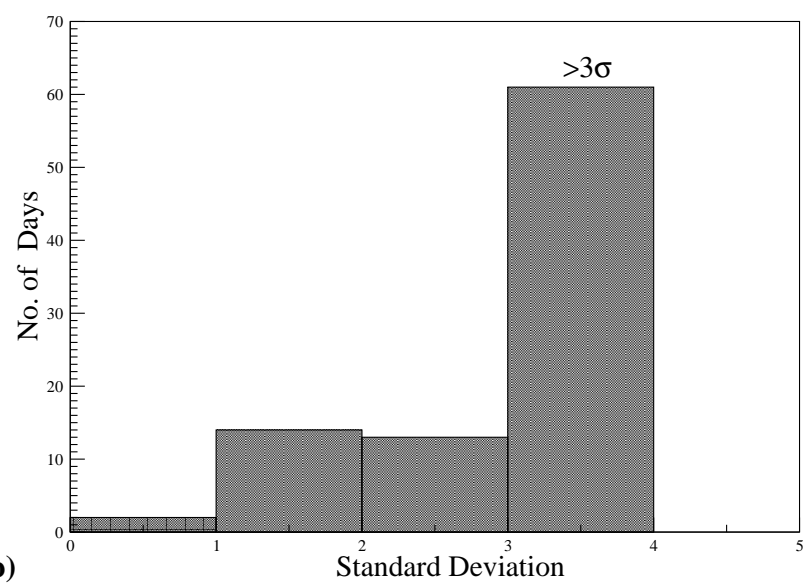

Fig. 4. The histograms showing the number of days in which (a) DLPT and (b) DLDT exhibited deviations. DLPT shows deviations above $3 \sigma$ for only 9 days, but DLDT shows such a deviation for more than 60 days.

deviations took place. For instance, DLPT shows deviations above $3 \sigma$ for only 9 days, but DLDT shows such a deviation for more than 60 days.

\section{Correlation of seismic activities with the VLF data}

In Paper 1, it was discussed that the terminator shifts may take place two days prior to the earthquakes. So it is pertinent to ask, if anomalies in DLDT and DLPT are also observed prior to the earthquakes or not. In order to give an example, we plot in Fig. 5 the amplitudes of the signal (shifted by $30 \mathrm{~dB}$ vertically for better viewing) for eleven consecutive days. On 22 January, there was an earthquake of a magnitude $M$ 6.0. In the data of 21 January, 2008, drawn here with thick curves, we clearly show that the signal near the sunrise terminator is totally different, and the normal sharp drop associated with the sunrise is replaced by a flatter variation. The signal behavior near the sun-set terminator is also different from those of the other days.

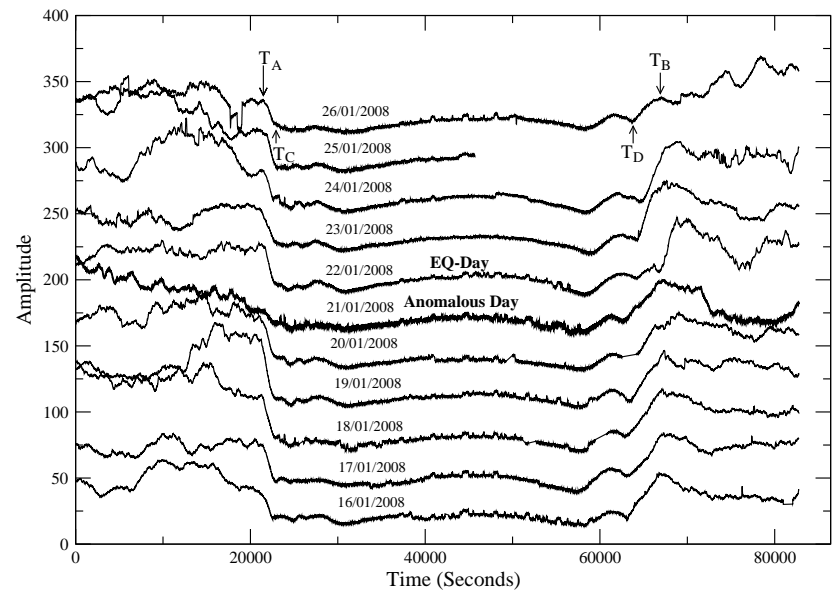

Fig. 5. The variation of the amplitudes of the VTX signal as a function of time in seconds for 11 consecutive days: 16 January 2008-26 January 2008. Days are stacked after an amplitude shift of 30 units. The points $\mathrm{T}_{\mathrm{A}}, \mathrm{T}_{\mathrm{C}}, \mathrm{T}_{\mathrm{D}}$ and $\mathrm{T}_{\mathrm{B}}$ are denoted in one of the days and DLPT $=\mathrm{T}_{\mathrm{C}}-\mathrm{T}_{\mathrm{A}}$ and DLDT $=\mathrm{T}_{\mathrm{B}}-\mathrm{T}_{\mathrm{D}}$. On 22 January 2008, an earthquake occurred with $M$ 6.0. The data on 21 January, plotted with a thick line, clearly shows that on 21 January 2008, the DLPT and the DLDT are anomalously higher than those of the other days. The signal also shows that before and after 5 to 6 days of the "earthquake day" the value of DLPT and DLDT is normal.

In Fig. 6, we plot DLPT and DLDT in minutes for all the strong earthquakes having a magnitude $M>5$ (upper two panels) which belong to the first three seismic circles only (i.e., a zone of radius $1500 \mathrm{~km}$ with centre at FRP). In all the cases, earthquakes take place on day " 0 ". It is clear that the DLPT and DLDT are generally higher prior to the seismic events and not after them. In fact, if we take simple averages of DLPT and DLDT, we find (third panel) that the average is peaked two days prior to the peak for DLPT and one day prior to the peak for DLDT. The error-bars are the standard deviations obtained on each day.

As in Paper 1, we plot Fig. 7a-b, the cross-correlations between the (a) DLPT or (b) DLDT and the effective magnitudes of the earthquakes which takes place on " 0 " day. Earthquakes of all the effective magnitudes were taken in this graph. In Fig. 7a, the peak occurred one day prior to the earthquake and there are also smaller peaks. In Fig. 7b, we note a quite broad peak, though it also occurred one day prior to the event day. In terms of predictability, we find that the terminator shift approach (Paper 1) gives indications of possible seismic events earlier than the DLDT or DLPT approach.

In Fig. 8a-d, we plot a similar result as in Fig. 7a-b, taking the depths of the seismic events into consideration. In (a) and (c) we plot the correlation coefficients for those quakes having shallow depths $(d<20 \mathrm{~km})$ and in (b) and (d) we consider those quakes having deeper depths $(d>20 \mathrm{~km})$. We generally find that the peak is sharper for shallower quakes. 

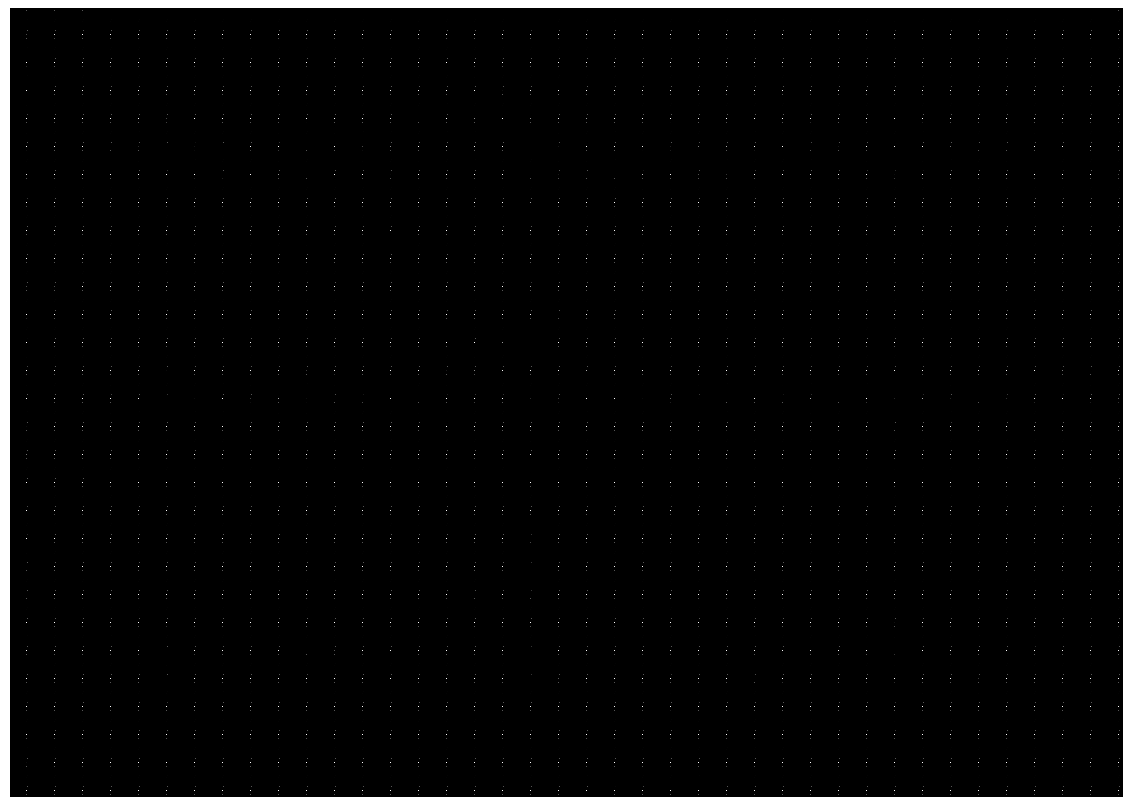

Fig. 6. The variation of the DLPT and DLDT as a function of days for a period of 15 days around the seismic events. The first panel shows the variation of the DLPT and the second panel shows the variation of the DLDT. The zero of the X-axis indicates the day of 14 earthquakes having $M>5$. The third panel shows the average variation of the DLPT (filled circles) and DLDT (filled squares) obtained from the first two panels with standard deviations as error bars for those 14 earthquakes. It is clear from the third panel that during the earthquakes, the value of DLPT is maximum on two days before the earthquakes and the value of the DLDT is maximum on one day before the earthquakes.
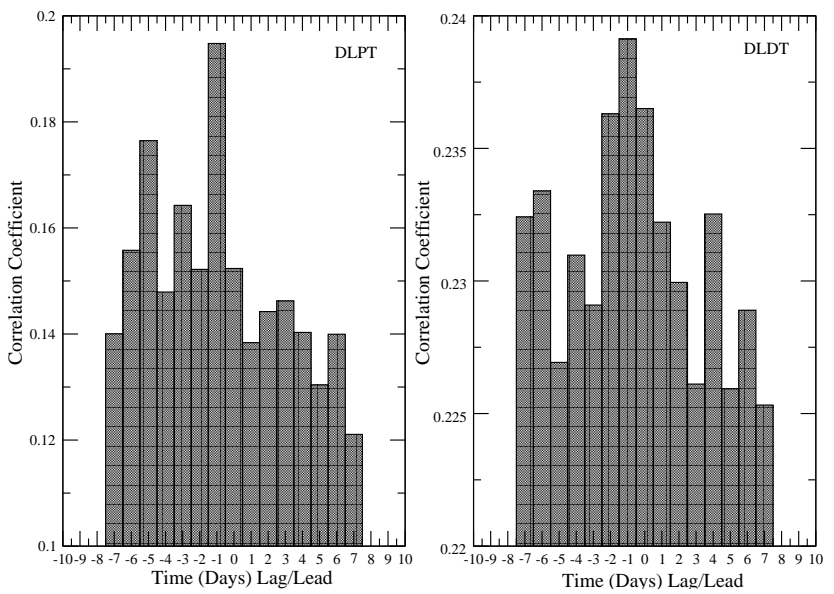

Fig. 7. The cross-correlations between the DLPT (a) or DLDT (b) with the effective earthquake magnitude occurs are plotted as a function of days before and after the event ("0"th day). In (a), the peak occurs one day prior to the seismic event but the effect continues even after the event. In (b), the peak is fairly broad, and appears at around $0.5-1$ day prior to the event.

In Fig. 9, we plot the effective magnitude of the earthquakes as a function of the standard deviation from the mean. The correlation is generally linear, i.e., on a given day, the effective magnitude or the energy deposited of the associated earthquake is directly related to the deviation of the DLPT (left) and DLDT (right).

\section{Concluding remarks}

It is long conjectured that ionospheric anomalies could have been detected prior to an earthquake. A number of groups have been working on this problem for last two decades and some evidences have been found. Since the Indian subcontinent is also vulnerable to severe earthquakes, we have been systematically recording the signals over the last few years to either establish or to refute the conjecture. In Paper 1 and in the present paper, we have used only the VTX data as received from Kolkata. In Paper 1, we concentrated on the calibration of the sunrise and sunset terminator times over the whole year so that anomalies may be studied easily. This was also possible because during 2005-2008, the sun was particularly quiet and there was no disturbances due to solar activities. There we showed that there is possibly a distinct signature of anomaly in the terminator timings and anomaly is seen almost $48 \mathrm{~h}$ prior to the seismic events. In the present paper, we chose an alternate measurable quantity, namely the D-layer preparation time (DLPT) and D-layer disappearance time (DLDT). We successfully demonstrated that the more the energy released due to seismic events on a given day is, the more is the deviation of DLPT and DLDT from the mean. However, the correlation is not very tight and as a result, the predictability is poorer. We showed that in both DLPT and DLDT, the cross-correlation is peaked only about a day prior to the seismic event. We used both the effective magnitude where we add the energy released from small quakes also, 

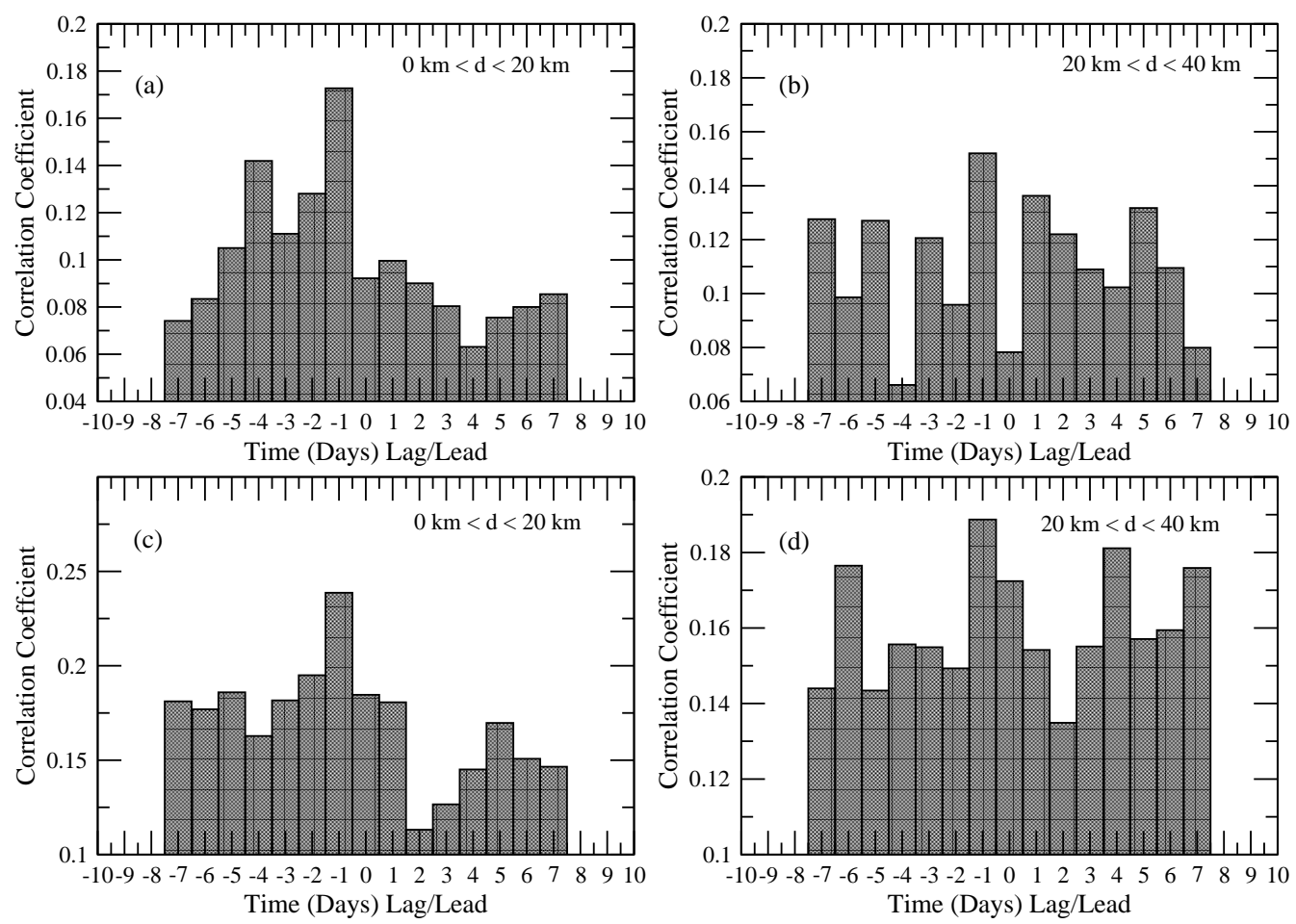

Fig. 8. The cross-correlations between the DLPT (a-b) or DLDT (c-d) with the effective earthquake magnitudes as a function of days before and after the event ("0"th day). (a) and (c) show the results for the earthquakes for which the depths $(d)$ of the epicenter are less than $20 \mathrm{~km}$ and (b) and (d) show the results of the earthquakes having depths of the epicenters between 20 and $40 \mathrm{~km}$. In all the cases, the peak occurs 1 day prior to the seismic event. For the shallow earthquakes $(d<20 \mathrm{~km})$ the peak is sharper and there are no other strong peaks before and after the event. For the deeper earthquakes $(20<d<40)$ there are smaller peaks at other days also.
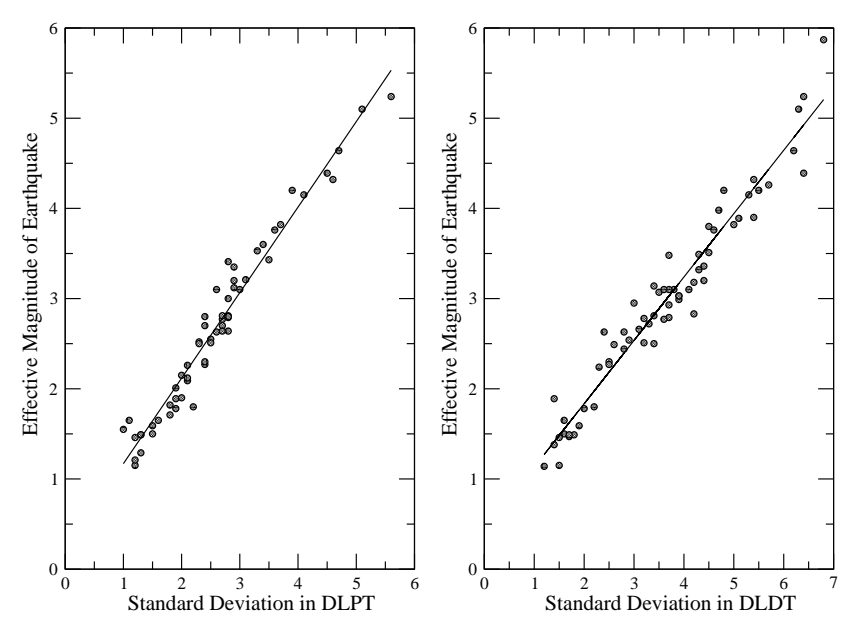

Fig. 9. Effective magnitudes of the earthquakes as a function of the deviations from the mean of DLPT (left) and DLDT (right). The correlation is generally linear, i.e., the effective magnitude or the energy deposited of the associated earthquake is directly related to the observed deviation. and the absolute magnitudes and showed that in both the cases the pre-cursors are present. What is more, we found that for shallower earthquakes $(d<20 \mathrm{~km})$ the correlation peaks are sharper than the quakes which occur at a higher depths $(d>40 \mathrm{~km})$.

Although VTX-ICSP baseline might have exhibited a correlation, the predictability of the actual event location is still not possible. We conjecture that if we carry out such observations from a multiple number of receiving stations, then the baseline exhibiting a tighter correlation is affected more by seismic events. Thus by taking data of multiple stations we will possibly be in a position to locate the region of seismic activities well ahead of time.

Acknowledgements. This project is supported by a RESPOND grant from ISRO.

Edited by: M. E. Contadakis

Reviewed by: three anonymous referees 


\section{References}

Chakrabarti, S., Sasmal, S., Saha, M., Khan, R., Bhowmik, D., and Chakrabarti, S. K.: Unusual behaviour of D-region Ionization time at $18.2 \mathrm{KHz}$ during seismically active days, Indian J. Phys., 81(5, 6), 531-538, 2007.

Clilverd, M. A., Rodger, C. J., and Thomson, N. R.: Investigating seismoionospheric effects on a long subionospheric path, J. Geophys. Res., 104(A12), 28171-28179, 1999a.

Clilverd, M. A., Rodger, C. J., and Thomson, N. R.: Sunrise effects on VLF signals propagating over a long north-south path, Radio Sci., 34(4), 939-948, 1999b.

Gokhberg et al.: Phys. Earth Planet. Inter., 57, 64-67, 1989.

Gufeld, I. L., Rozhnoi, A. A., Tyumensev, S. N., et al.: Radiowave disturbances in period to Rudber and Rachinsk earthquakes, Phys. Solid Earth, 28(3), 267-270, 1992.

Lowrie, W.: Fundamentals of Geophysics, Cambridge University Press, UK, 2007.

Hayakawa, M., Molchanov, O. A., Shima, N., Shvets, A. V., and Yamamoto, N.: Seismo Electromagnetics: Lithosphere Atmosphere-Ionosphere Couplings, edited by: Hayakawa, M. and Molchanov, O. A., TERRAPUB, Tokyo, Japan, 223 pp., 2003.

Maekawa, S., Horie, T., Yamauchi, T., Sawaya, T., Ishikawa, M., Hayakawa, M., and Sasaki, H.: A statistical study on the effect of earthquakes on the ionosphere, based on the subionospheric LF propagation data in Japan, Ann. Geophys., 24, 2219-2225, doi:10.5194/angeo-24-2219-2006, 2006.
Molchanov, O. A. and Hayakawa, M.: Subionospheric VLF signal perturbations possibly related to earthquakes, J. Geophys. Res, 103, 17489-17510, 1998.

Molchanov, O. A., Hayakawa, M., Ondoh, T., and Kawai, E.: Precursory effects in the subionospheric VLF Signals for the Kobe earthquake, Phys. Earth Planet. In., 105, 239-248, 1998.

Rodger, C. J., Clilverd, M. A., and Thomson, N. R.: Modeling of subionospheric VLF signal perturbations associated with earthquakes, Radio Sci., 34(5), 1177-1185, 1999.

Rozhnoi, A., Solovieva, M., Molchanov, O., Schwingenschuh, K., Boudjada, M., Biagi, P. F., Maggipinto, T., Castellana, L., Ermini, A., and Hayakawa, M.: Anomalies in VLF radio signals prior the Abruzzo earthquake $(M=6.3)$ on 6 April 2009, Nat. Hazards Earth Syst. Sci., 9, 1727-1732, doi:10.5194/nhess-91727-2009, 2009.

Sasmal, S. and Chakrabarti, S. K.: Ionosperic anomaly due to seismic activities - Part 1: Calibration of the VLF signal of VTX $18.2 \mathrm{KHz}$ station from Kolkata and deviation during seismic events, Nat. Hazards Earth Syst. Sci., 9, 1403-1408, doi:10.5194/nhess-9-1403-2009, 2009.

Soloviev, O. V. and Hayakawa, M.: Three-dimensional subionospheric VLF field diffraction by a truncated highly conducting cylinder and its application to the Trimpi effect problem, Radio Sci., 37, 1079, doi:10.1029/2001RS002499, 2002.

Soloviev, O. V., Hayakawa, M., Ivanov, V. I., and Molchanov, O. A.: Seismo-electromagnetic phenomenon in the atmosphere in terms of 3D subionospheric radio wave propagation problem, Phys. Chem. Earth, 29, 639-647, 2004. 